Article

\title{
A Compact Component for Multi-Band Rejection and Frequency Coding in the Plasmonic Circuit at Microwave Frequencies
}

\author{
Wenxuan Tang ${ }^{1,2} \mathbb{D}^{\text {, Yujie Hua }}{ }^{1}$ and Tie Jun Cui ${ }^{1,2, *}$ \\ 1 State Key Laboratory of Millimeter Waves, School of Information Science and Engineering, \\ Southeast University, Nanjing 210096, China; wenxuant@seu.edu.cn (W.T.); 220200839@seu.edu.cn (Y.H.) \\ 2 Institute of Electromagnetic Space, Southeast University, Nanjing 210096, China \\ * Correspondence: tjcui@seu.edu.cn
}

Citation: Tang, W.; Hua, Y.; Cui, T.J. A Compact Component for Multi-Band Rejection and Frequency Coding in the Plasmonic Circuit at Microwave Frequencies. Electronics 2021, 10, 4. https://dx.doi.org/10.3390/electronics 10010004

Received: 17 November 2020 Accepted: 18 December 2020 Published: 23 December 2020

Publisher's Note: MDPI stays neutral with regard to jurisdictional claims in published maps and institutional affiliations.

Copyright: () 2020 by the authors. Licensee MDPI, Basel, Switzerland. This article is an open access article distributed under the terms and conditions of the Creative Commons Attribution (CC BY) license (https: / creativecommons.org/ licenses/by/4.0/).

\begin{abstract}
Plasmonic circuits, which support the propagation of spoof surface plasmon polaritons (SSPPs) at microwave frequencies, have been developed in recent years as an expected candidate for future highly integrated systems, mainly because of their extraordinary field confinements and sub-wavelength resolution. On the other hand, artificial electromagnetic (EM) resonators are widely adopted in metamaterial design for flexible resonance and band gaps. In this work, an electrically small complementary spiral, which is made up of six helix branches sculptured in the ground, is proposed to achieve independent resonances at six different frequency bands. Combined with the grounded corrugated transmission line (TL), the proposed component can provide designable multi-band rejection, and compose frequency coding circuits with a compact size (less than $\lambda_{0} / 4$ ). The complementary spirals excited with the bending TL and the straight one are both investigated, and independence band rejections and designed 6-bit coding sequences in the frequency spectrum are demonstrated numerically and experimentally. Hence, it is concluded that such compact components can be adopted to flexibly control the rejection of waves in multi-frequency bands, and benefits the development of frequency-identification circuits and systems.
\end{abstract}

Keywords: band rejection; spoof surface plasmon polaritons; complementary resonator; compact circuit; coding metamaterials

\section{Introduction}

Metamaterials have been developing dramatically since the end of the last century, when Sir John Pendry et al. proposed the schemes of realizing negative permittivity $(\varepsilon)$ and permeability $(\mu)$ through arrays of resonant unit cells $[1,2]$. The electrically small unit cells, such as the split ring resonators (SRRs), the electric-LC (ELC) resonators [3], and the I-shaped structures [4], have been demonstrated as the composing "atoms" for the artificial metamaterials. Such atoms respond to outer electromagnetic (EM) waves, and possess a real part of permittivity (for electric resonance) or permeability (for magnetic resonance) that follows the Lorentz model, and therefore can be flexibly designed to present the required EM parameters from the point of view of effective medium theory [5]. Bulky metamaterials have been realized using dielectric or metallic resonating unit cells assembled periodically, and attractive applications such as invisible cloaking and gradient index lenses have been delivered [6-10]. Furthermore, the complementary SRR (CSRR), which is a metallic screen with the negative image of SRR, was developed as the dual counterpart of SRR [11]. Based on the Babinet principle, there is a duality for the complementary structure [12]. For example, when the SRR performs as a magnetic resonator, the CSRR performs as an electric one. The complementary resonators are especially applicable in planar circuits, as they can be sculptured in the ground without binging in extra space. 
In recent years, metasurfaces, the planar version of metamaterials, have received great interest for their low profile, novel functionality, and easy integration [13,14]. In addition, instead of using effective medium parameters to describe metamaterials, digitally coding metamaterials have been developed to build a novel connection between the digital world and the physical one $[15,16]$. The incident EM waves can be tailored digitally by establishing different coding sequences composed of " 0 " and " 1 ", which represent phase or amplitude information in a unit or unit cells. Phase codes and amplitude codes have been adopted in digital metasurfaces to perform new-concept EM information manipulations and processing $[17,18]$.

Most recently, a special type of two-dimensional (2D) metamaterials, the plasmonic metamaterials, were created to support the propagation of spoof surface plasmon polaritons (SSPPs). The surface plasmon polaritons (SPPs) are highly localized surface waves existing on the interface between two media with opposite permittivity [19]. They do not exist in nature below the optical frequency. At terahertz and microwave frequencies, however, decorations such as periodic hollows and corrugations are added on metal surfaces to pave a way for "spoof" surface plasmon polaritons [20]. Similar to the SPPs, the SSPPs possess remarkable properties such as extraordinary field confinements and sub-wavelength resolution. The SSPPs have been realized and verified on structured metal surfaces, corrugated semiconductors, corrugated transparent conducting oxides, et al., for different purpose [21-27]. For microwave circuits, a type of planar SSPP waveguide, the SSPP transmission lines (TLs) printed on ultrathin dielectric substrates, were delivered and investigated [28]. Thanks to the above-mentioned unique properties, the SSPP TLs exhibit controllable dispersion characteristics, strong field restriction, depressed mutual coupling, low cross talks, and low radiation loss [29-32], and hence have great potentials in integrated circuits and highly compact systems [33-35].

In the circuit, the SSPP mode is supported in single-conductor TL, where mode conversion from guided-wave mode (e.g., the quasi-transverse electromagnetic (TEM) mode in the coplanar waveguide (CPW) or the microstrip (MS) line) is necessary [36,37]. In addition, considering the environment in compact planar systems, e.g., in the packaged integrated circuits and in the CMOS, metallic ground exists when needed. The grounded SSPP TL includes the ground plane on the bottom of the dielectric together with the corrugated strip on the top, and therefore is the same as the loaded microstrip line in microwaves [38]. We denote that since the periodic corrugation is subwavelength and the SSPP TL is derived from corrugated surfaces by a metamaterial that hosts an effective plasma frequency, it can also be analyzed from the point of view of metamaterials [39]. Due to the existence of the ground plane, the EM field is severely restricted as that of the quasi-TEM mode; however, a nonnegligible portion of the electric field is still maintained between neighboring corrugations, resulting in a longitudinal electric field in the propagation direction and energy localization in the transverse plane $[40,41]$. In view of this, the grounded corrugated TLs support the hybrid mode containing the quasi-TEM mode and the SSPP one, and can be adopted in plasmonic circuits [40-43]. They are well compatible for integrated circuits, and have potentials for bringing in compact components [44] and depressing crosstalks [45].

In this paper, we firstly propose a sub-wavelength scaled complementary resonator, which is a metallic screen with the negative image of a central-symmetry spiral which includes six helix branches. By embedding different gaps in different branches, we are able to create six different resonances independently. After that, we design a compact section of grounded SSPP TL and added the complementary resonator on the ground right below the TL. We analyze and demonstrate that each branch can create a band rejection independently, and the resonator in total can create six band rejections. The multi-band rejection could also be considered as a kind of digital coding in the frequency domain. In other words, the proposed complementary spiral can produce 6-bit frequency code for the plasmonic circuits and systems whilst occupying a space sized less than $\lambda_{0} / 4 \diamond \lambda_{0} / 4$. In the end, coding components excited by the bending SSPP TL and the straight SSPP TL are designed and fabricated, and multi-band rejections and different coding sequences 
are demonstrated in experiment. Discussions are performed and conclusions are drawn according to the results.

\section{Design of the Compact Component}

\subsection{The Complemantary Six-Branch Spiral Resonator}

For an electric or magnetic resonator, induced EM fields are aroused by outer EM waves at a specific frequency, and resonance happens accordingly. The sub-wavelength scaled cut-wire and the I-shaped structure (in Figure 1a) are the firstly used electric resonators for metamaterials [4]. When the electric field of an incident wave is parallel to the longitudinal wire, induced currents flow on the wire and electric resonance therefore happens. On the other hand, the SRR (in Figure $1 \mathrm{~b}$ ) is the firstly proposed magnetic resonator [2]. When the magnetic field of incident wave is normal to the surface of the SRR, induced currents flow in the ring and magnetic resonance therefore happens. When the resonance is strong enough, negative real part of permittivity or permeability appears according to the Lorentz-Drude dispersion curve of materials [5]. In addition, other kinds of resonators, such as the ELC resonator [3] (in Figure 1c) and the spiral resonator (in Figure 1d) [46], have also been conceived and demonstrated.

\section{(a)}

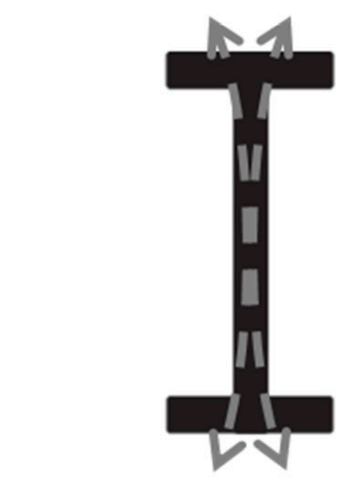

\section{(c)}

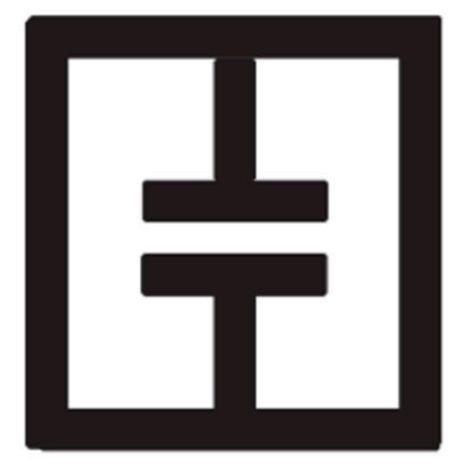

(b)

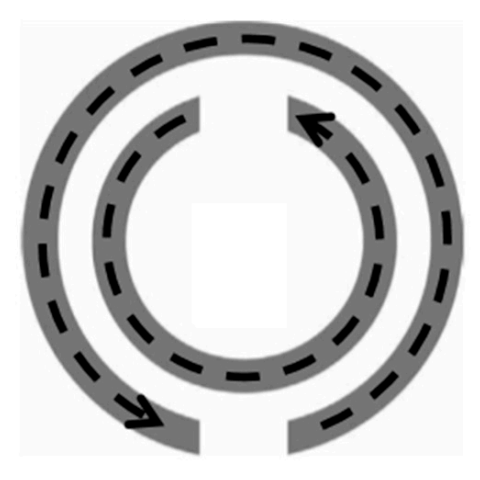

(d)

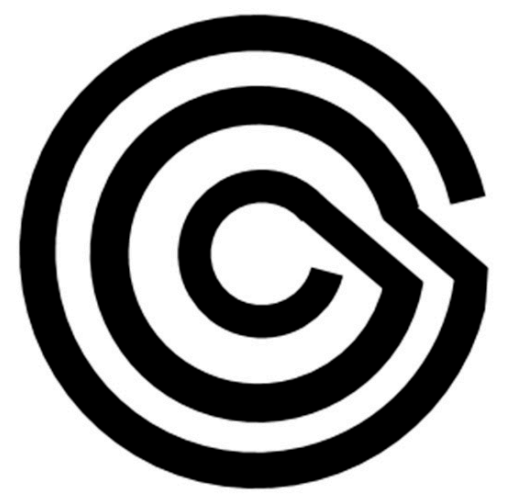

Figure 1. Several types of the electromagnetic (EM) resonators for metamaterials. (a) The I-shaped resonator. (b) The split ring resonator. (c) The electric-LC resonator. (d) The spiral resonator. The dashed arrowed lines indicate the resonating currents.

It is noted that the electric resonance and the magnetic one may happen in the same resonator [47]. For example, a spiral serves as a magnetic resonator when the external magnetic field is aligned along the axes of the "loop" of the induced currents, or an electric one when the external electric field is aligned along a section of the induced currents. However, the above-mentioned structures respond to the outer EM waves as single resonators. Although high-order resonances exist in the higher spectrum, their frequencies are usually several times of that for the fundamental mode. When the fundamental resonance is tuned through the geometric parameters, the higher ones change as well. In other words, frequencies of different orders of resonance are usually related to each other, and one is not able to independently tune a single resonance in the spectrum. 
In view of this, we present a central-symmetry spiral which includes six helix branches (as is given in Figure 2a). The six branches can respond to the outer EM wave and resonate all together, as well as independently. Furthermore, if different branches are cut at different lengths, the lengths of induced currents become different as well, and each branch resonates at a unique frequency. In this way, one is able to create six different and independently controlled resonances using a single sub-wavelength spiral. On the other hand, the counterpart of the six-branch spiral, the complementary six-branch spiral as shown in Figure 2b, also provides six independent resonances. It is actually a metallic screen with the negative image of the spiral. According to the Babinet principle, there is a duality for the complementary structure, and the electric and magnetic fields of the resonators are interchanged [12].

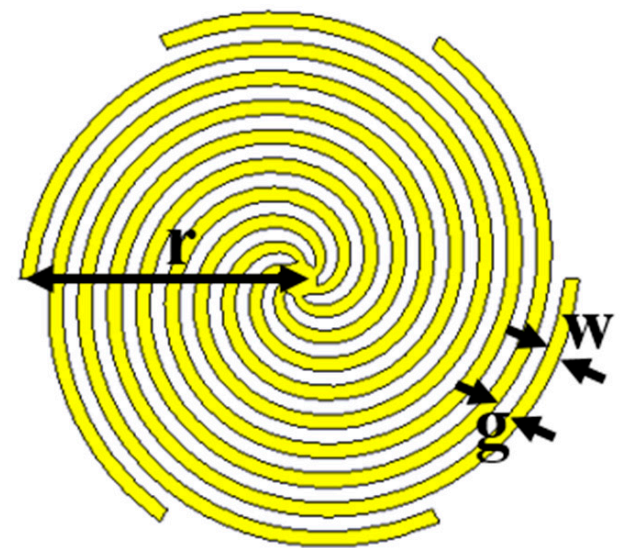

(a)

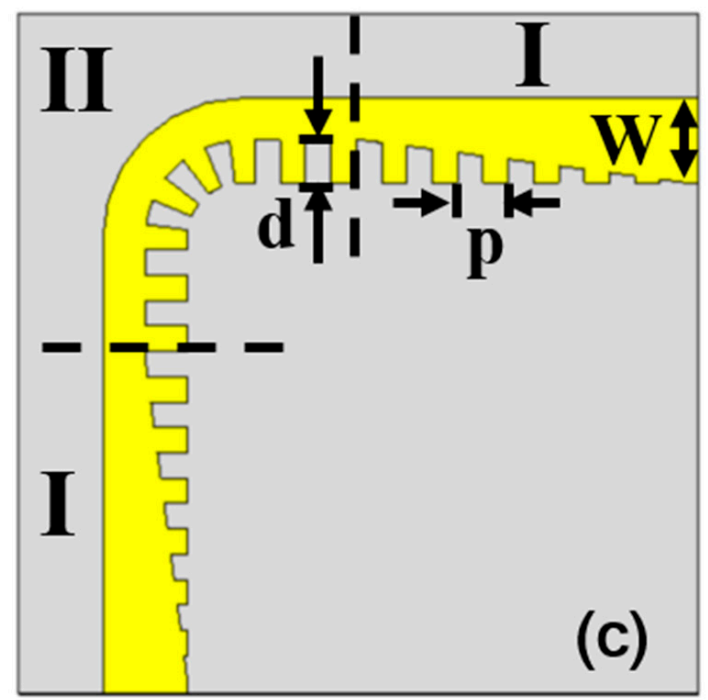

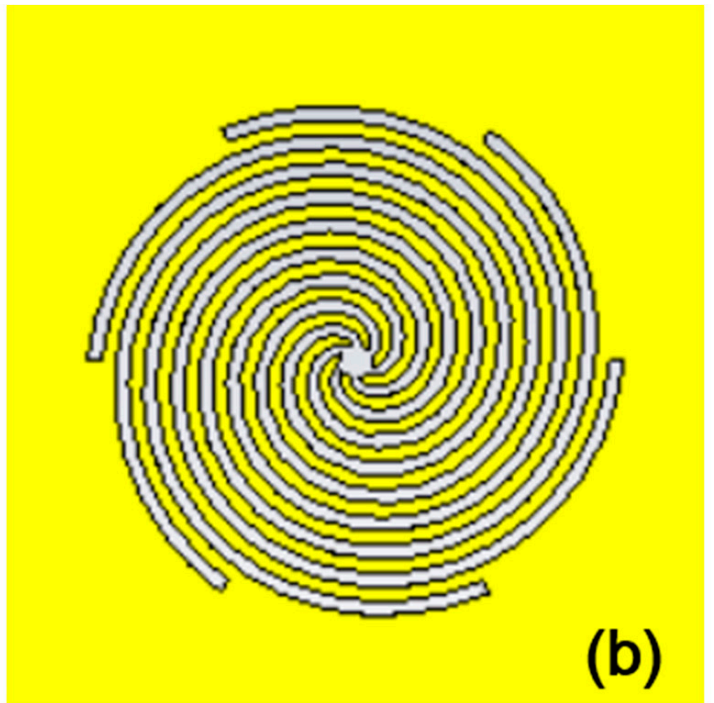

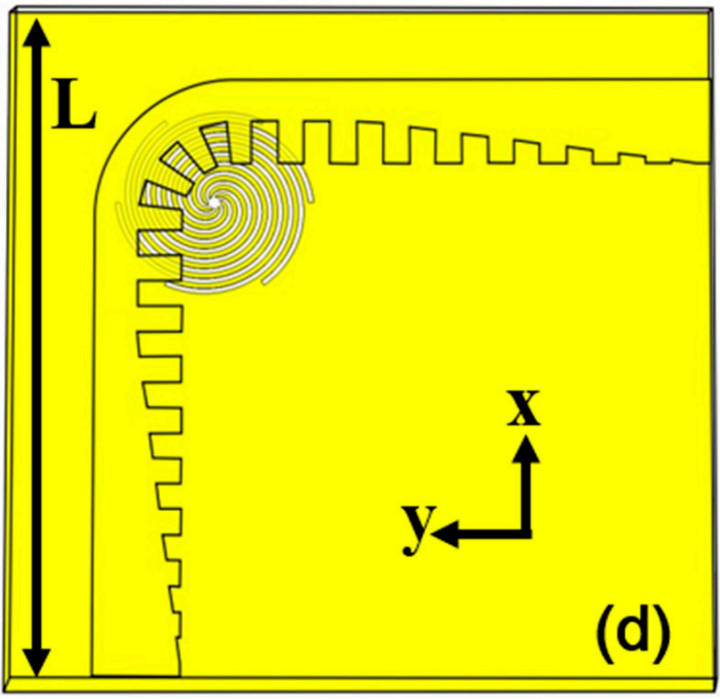

Figure 2. (a) The six-branch spiral resonator. (b) The complementary six-branch spiral resonator. (c) (Top view) The bending spoof surface plasmon polaritons (SSPP) transmission line. (d) (Perspective top view) The bending SSPP transmission line with the spiral resonator sculptured in the ground. Substrate is perspective in (d). The metal is illustrated in yellow, and the substrate in grey.

The proposed six-branch spiral resonators can be excited through the coupling of EM fields in transmission lines. In particular, the complementary spiral resonator is extremely applicable in planar circuits because it can be sculptured in the ground without bringing in extra space. In this work, we choose to use the grounded corrugated TL to 
excite the resonator. The reason why we did not use the microstrip line is given in the section of Discussion. To demonstrate the merit of field localization of SSPPs, both straight grounded corrugated TL and the bending one are designed, among which the bending one is depicted in Figure 2c for detail. The yellow part represents the copper strip, and the grey one presents the dielectric substrate of Rogers 5880 with permittivity of 2.2 , loss tangent of 0.0009 , and thickness of $1.575 \mathrm{~mm}$. The complementary spiral, whose diameter is $11.4 \mathrm{~mm}$, is located right below the bending part (please refer to the perspective top view in Figure 2d). Input and output are located in Section 1, where microstrip lines are connected to the grounded corrugated TL through gradient transition, and vice versa [40]. Section 2 is the grounded SSPP TL composed of comb-shaped unit cells. The characteristics of the bending TL are mainly determined by geometric dimensions, such as the periodic length $p$ and the depth of the comb $d$ (as denoted in Figure 2), and the modified parameters are listed in Table 1.

Table 1. Dimensions in Figure 2.

\begin{tabular}{ccc}
\hline Parameter & Value $(\mathbf{m m})$ & Description \\
\hline $\mathrm{L}$ & 30.23 & Size of the bending grounded SSPP TL (transmission line) \\
$\mathrm{W}$ & 5 & Width of the microstrip line at the input/output \\
$\mathrm{p}$ & 3 & Period length of the grounded SSPP TL \\
$\mathrm{d}$ & 2.5 & Depth of the comb \\
$\mathrm{r}$ & 5.7 & Radius of the spiral \\
$\mathrm{w}$ & 0.3 & Width of the branch \\
$\mathrm{g}$ & 0.3 & Width of the gap between neighboring branches
\end{tabular}

The dispersion characteristics are investigated during the design of the grounded SSPP TL. A comb-shaped unit cell in the free space (with the substrate and the ground) is modeled in the commercial software of CST Microwave Studio with a pair of Floquet periodic boundaries set in the propagation direction. The real part of the propagation constant is calculated via an eigenfrequency solver. Dispersion curves of the grounded SSPP TLs with different values of $d$ are plotted in Figure 3a and compared with that of the microstrip line. It is observed that as $d$ increases from zero (which is the case for the microstrip line), the dispersion curve gradually departs to the right side and the cut-off frequency of the SSPPs decreases in the meantime. That is to say, the TEM wave in the microstrip line is converted to the hybrid slow wave through the transition units with gradient depths $d$. Thanks to the smooth transition, impedance matching and momentum matching are realized to guarantee the efficiency. The imaginary part of the propagation constant is also calculated for the TL, with $d=2.5 \mathrm{~mm}$ using the method based on circuit topology [48], and the corresponding loss curve is plotted by the dashed black line in Figure 3a. It is observed that the loss in the uniform corrugated TL (without bending or transition sections) is pretty low below the cut-off frequency. In addition, it has also been demonstrated that the grounded SSPP TL possesses low bending loss and radiation loss [49]. Therefore, the bending SSPP TL without the complementary spiral in the ground, which is $30.23 \times 30.23 \mathrm{~mm}^{2}$ in total, possesses high transmission coefficient (S21) and low reflection coefficient (S11) below $10 \mathrm{GHz}$, as is demonstrated in Figure 3b. We denote that in this case the loss has mainly caused in the transitions and the bending section. 

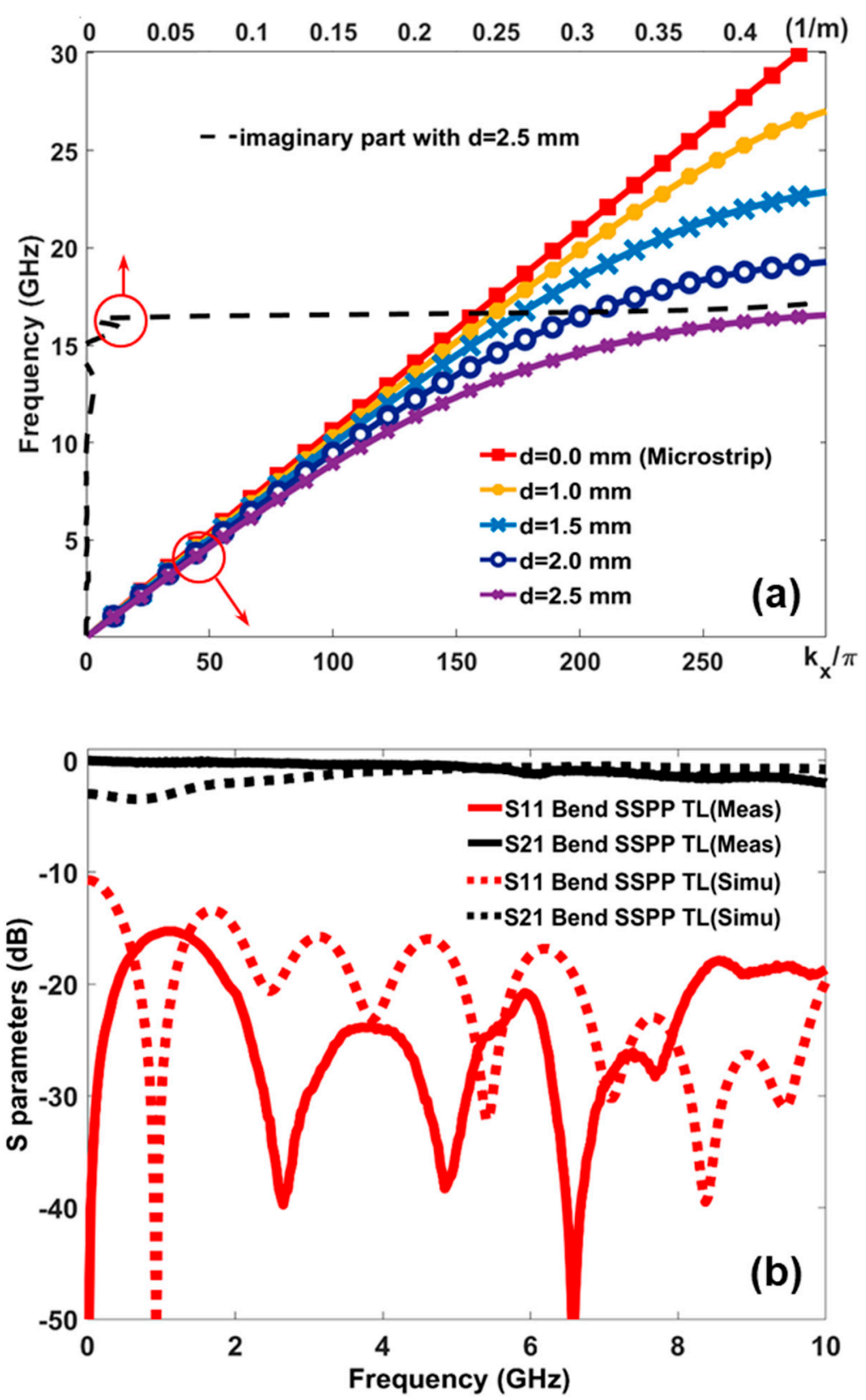

Figure 3. (a) Dispersion curves of the grounded SSPP TLs and the microstrip line, and the loss curve of the adopted SSPP TL. (b) The measured and simulated transmission coefficients (S21) and reflection coefficients (S11) of the bending SSPP TL without the complementary spiral in the ground.

\subsection{Multi-Band Rejection and 6-Bit Frequency Code}

When a metallic gap exists in the branch of the complementary spiral, the "gap" in fact is a metallic block inserted in the hollowed-out branch. Induced currents in this branch are blocked by it, and a corresponding resonance appears. For example, in the top left picture in Figure 4, a gap (numbered \#2) is added in one branch, and a resonance appears at $4.06 \mathrm{GHz}$. Detailed distribution of the EM fields and currents at $4.06 \mathrm{GHz}$ is depicted in Figure 5. It is observed in Figure 5a that the EM energy is coupled from the corrugated strip (located in the dashed blue line) to the spiral in the ground (located in the dashed black line), and the electric field in the spiral is resonating. The magnitude of the electric field in Figures 4 and $5 b$ indicate that very limited energy is coupled to other branches. Induced currents in the spiral are also depicted in Figure 5c, showing that the currents terminate at the gap, and the resonance is excited accordingly. 


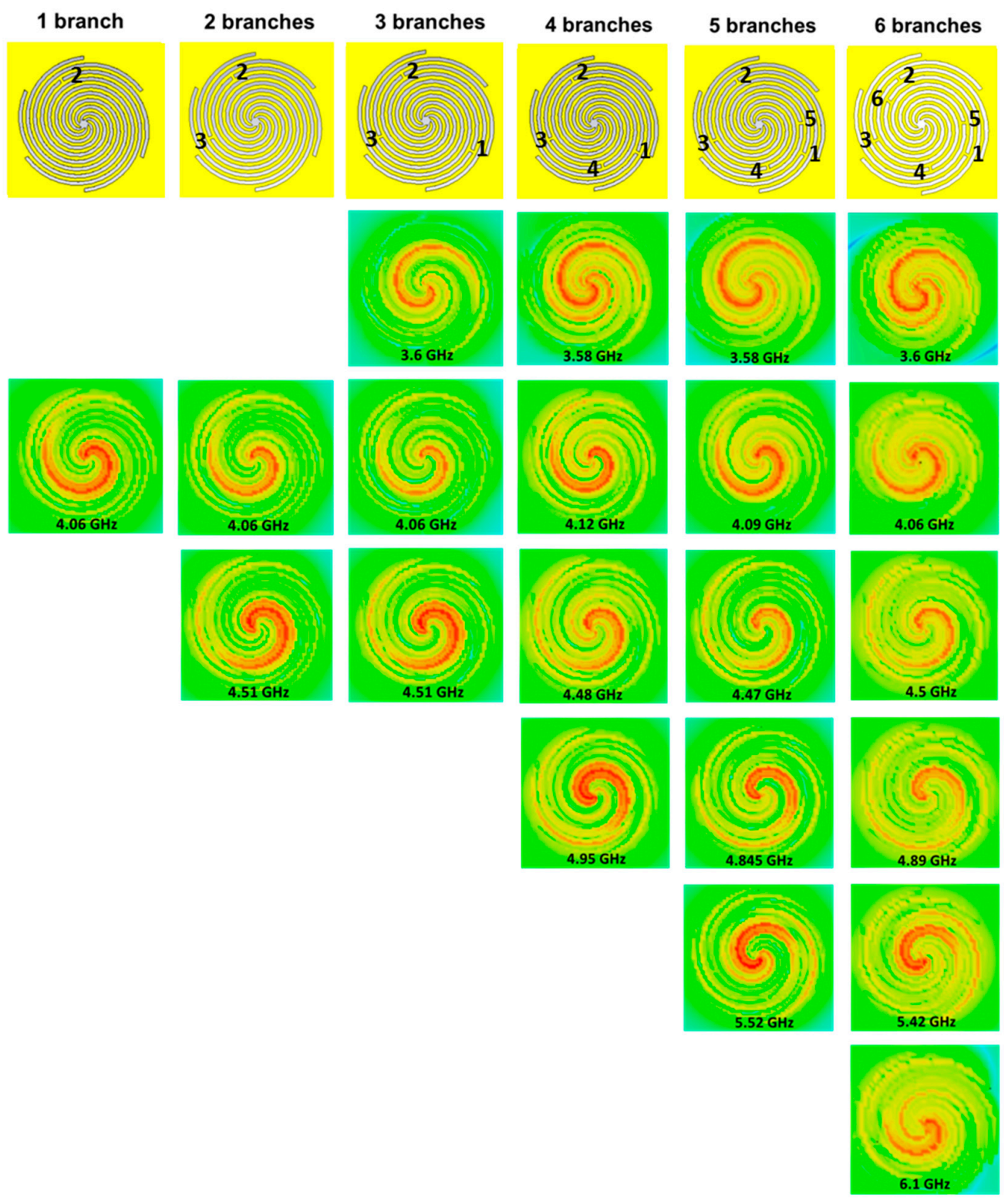

Figure 4. Normalized magnitudes of the electric fields at resonance frequencies when different numbers of gaps (1 to 6 from left to right) are included in the complementary spiral resonator.
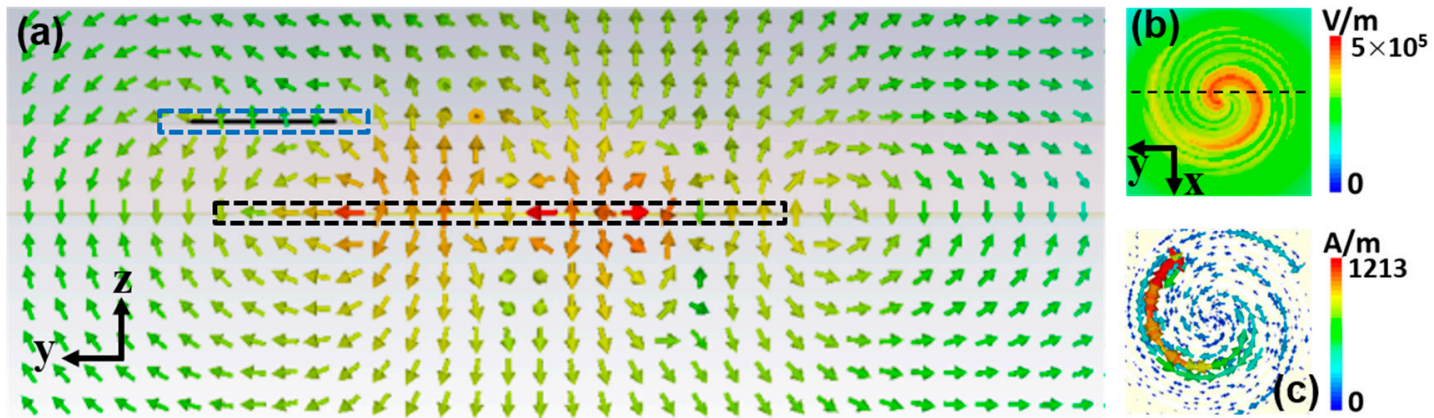

Figure 5. Electromagnetic fields and currents at $4.06 \mathrm{GHz}$. (a) (Side view) Distribution of the electric field. The spiral resonator locates in the dashed black line and the corrugated strip in the dashed blue line. (b) (Top view) Magnitude of the electric field. (c) (Top view) Distribution of currents in the resonator. 
When two gaps are added respectively in two different branches, as is illustrated in the second column of Figure 4, two resonances appear at 4.06 and $4.51 \mathrm{GHz}$, corresponding to the \#2 and \#3 gaps, respectively. It is observed that, for the 2-branch case, the magnitude of the electric field at $4.06 \mathrm{GHz}$ remains nearly unchanged when compared to that for the 1-branch case, whilst at $4.51 \mathrm{GHz}$, a new resonance appears in the other branch. Furthermore, when more gaps are added in different branches, more resonances are detected at different frequencies. Figure 4 reveals that the resonance in one branch is not influenced by other branches in terms of field distribution and resonance frequency. That is to say, the complementary six-branch spiral can provide six independent resonances in maximum. We denote that the gaps in Figure 4 are numbered from 1 to 6, where Gap \#1 is related to the resonance at the lowest frequency, and Gap \#6 is related to that at the highest frequency.

The six complementary spiral resonators listed in the first line of Figure 4 are adopted on the bottom side of the bending SSPP TL and excited by the strong near field coupling. Multi-band rejections are observed due to the existence of resonances. Figure 6 plots the transmission coefficients for the six cases when gaps are added to one branch, two branches, three branches, et al. The dashed black curve shows the simulated S21 for the case when only one branch contains a gap (Gap \#2), and a $-22.17 \mathrm{~dB}$ band rejection is observed at $4.06 \mathrm{GHz}$. The blue curve with hollow circles contains two band rejections centered at 4.06 and $4.51 \mathrm{GHz}$, and the lowest transmission coefficients are $-21.77 \mathrm{~dB}$ and $-17.15 \mathrm{~dB}$, respectively. When there are three branches and more, the number of band rejections increases accordingly. Center frequencies and valley values of the band rejections are listed in Table 2 for details. It is remarkable that the center frequencies of band rejections are in accordance with the resonance frequencies in Figure 4, proving that the band rejections are indeed caused by the resonances in the branches. The rejections at $4.06,4.50$, and $4.89 \mathrm{GHz}$ have the valley value of S21 below $-20 \mathrm{~dB}$, whilst the other three rejections are also significant enough to test. In addition, each branch is resonating in a specific frequency that is determined by the position of its gap, and the resonance frequency for each branch does not significantly shift when neighboring gaps appear or disappear. In other words, the band rejections are independent of each other.

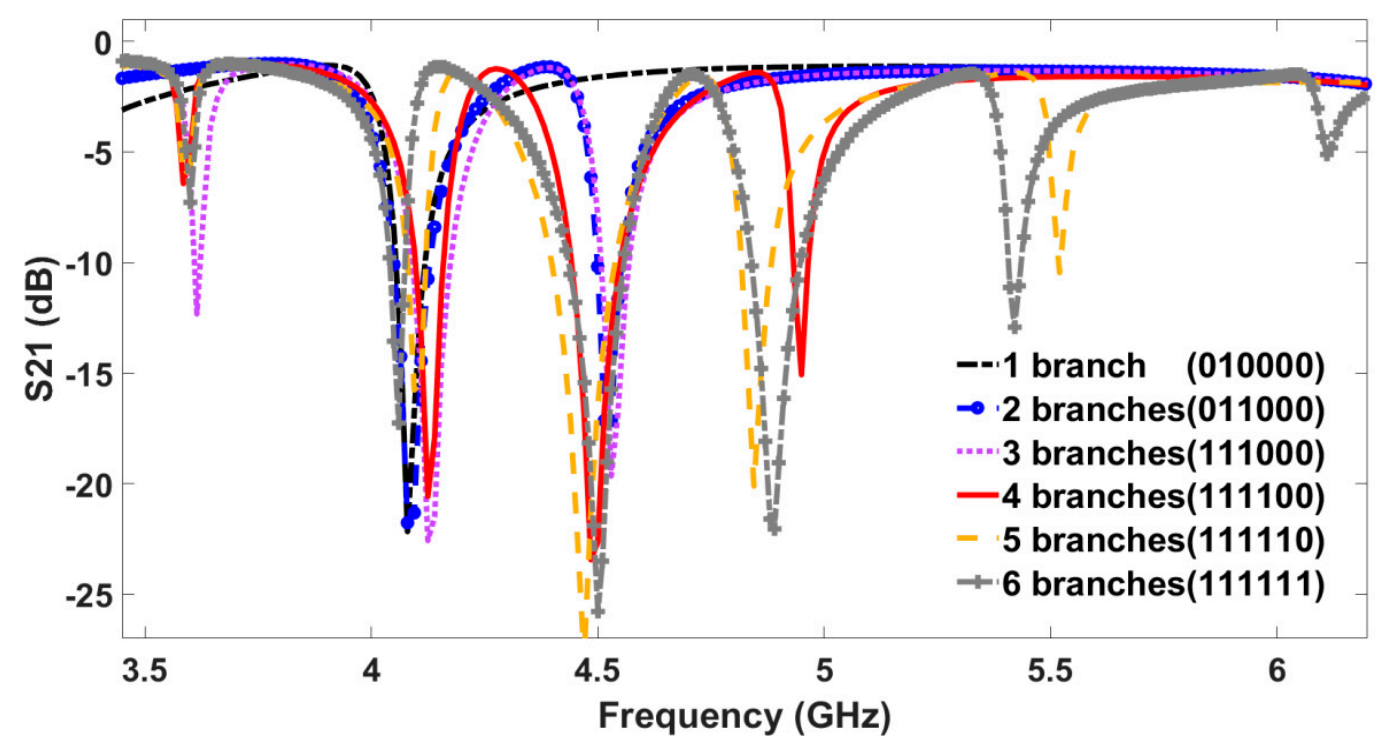

Figure 6. The transmission coefficients (S21) when a different numbers of branches have a gap. From the point of view of digital coding metamaterials, the band rejections can be considered as a type of frequency code. 
Table 2. Band rejections with different types of complementary spiral. The spiral types are defined in Figure 4.

\begin{tabular}{ccc}
\hline Spiral Type & Center Frequency of Band Rejection (GHz) & Valley Value of S21 (dB) \\
\hline 1 branch & 4.06 & -22.17 \\
2 branches & $4.06,4.51$ & $-21.77,-17.15$ \\
3 branches & $3.60,4.06,4.51$ & $-12.33,-22.58,-19.66$ \\
4 branches & $3.58,4.12,4.48,4.95$ & $-6.44,-20.58,-23.45,-15.08$ \\
5 branches & $3.58,4.09,4.47,4.85,5.52$ & $-6.44,-15.77,-27.88,-20.12,-10.46$ \\
6 branches & $3.60,4.06,4.50,4.89,5.42,6.10$ & $-7.27,-17.27,-25.78,-22.03,-12.92,-5.12$ \\
\hline
\end{tabular}

From the point of view of digital coding metamaterials, the multi-band rejection can be adopted to realize a new type of frequency code, aside from the phase codes and amplitude codes. Although the commonly defined frequency coding is the frequency-shift keying (FSK), the band-pass property and band-rejection property in the frequency domain can also be represented by " 0 " and " 1 " in binary, respectively. This kind of frequency code has wide potentials in frequency-identification circuits and systems, such as the radio frequency identification devices (RFIDs). The above designed complementary spiral resonator is therefore applied to realize a compact 6-bit coding component, where each branch is responsible for one bit. Band rejection at the lowest frequency $(3.60 \mathrm{GHz}$ in this design) determines the highest bit of the coding sequence, whilst the one at the highest frequency $(6.10 \mathrm{GHz}$ in this design) determines the lowest bit. In particular, "1" represents the existence of the corresponding band rejection, and " 0 " represents the inexistence of the corresponding band rejection. For example, for the 1-branch case, only the band rejection at $4.06 \mathrm{GHz}$ (which is the second lowest frequency) exists, and therefore its frequency code is " 010000 ". Additionally, for the 3-branch case, there are three band rejections at 3.60, 4.06, and $4.51 \mathrm{GHz}$, and the frequency code becomes "111000". Some other examples of the frequency code are presented in Figure 6 as well. Theoretically, a spiral with $N$ branches can create $2^{N}$ frequency codes. On the other hand, when the transmission coefficient of the SSPP circuit is tested, the type and geometry of the resonator in the ground can be estimated.

\section{Experimental Results}

Prototypes of the above designed grounded SSPP TLs, including both the bending and the straight ones, are fabricated, and the complementary spirals are sculptured on the ground. Top views of the prototype are inserted in Figures 7 and 8, and the spirals are located inside the dashed orange circles on the bottom side. Transmission coefficients are measured using an Agilent vector network analyzer (VNA) and microwave cables and SMA connectors are used to connect the input and output to the two ports of the VNA. Four different complementary spirals with different coding sequences of 010101, 111111, 101001, and 101010 are adopted in the bending SSPP TL, and five different complementary spirals with different coding sequences of 000000, 010000, 010100, 111100, and 111110 are adopted in the straight SSPP TL in the measurement. We note here that, for example, 101001 is for the complementary spiral containing Gap \#1, Gap \#3, and Gap \#6 that were defined in the first line of Figure 4.

Band rejections are observed around 3.75, 4.13, 4.63, 5.07, 5.66, and $6.13 \mathrm{GHz}$ in measurement and presented in Figure 7. Compared with the center frequencies listed in Table 2, the measured frequencies are a bit higher than the simulated ones. This blue shift may be caused by the inaccuracy during fabrication and assembly. Nevertheless, the resonance in each branch has been proven to be strong enough and independent of each other, and the multi-band rejections are deep enough to be recognized and distinguished. Although the center frequency of band rejection may vary slightly with different spirals, e.g., the center frequency of the 4th band rejection is $5.07 \mathrm{GHz}$ for the 010101 spiral but changes to $4.94 \mathrm{GHz}$ for the 111111 spiral, this band rejection is still recognizable. In view of this, the frequency domain could be discretized and categorized into six rejection bands, as illustrated by the grey blocks in Figure 7 . When band rejection happens in a grey block, the corresponding 
bit is set to " 1 ", whilst when there is no band rejection in the grey block, the corresponding bit is set to " 0 ". In this way, the four coding sequences have been correctly detected and plotted in the frequency spectrum in Figure 7.

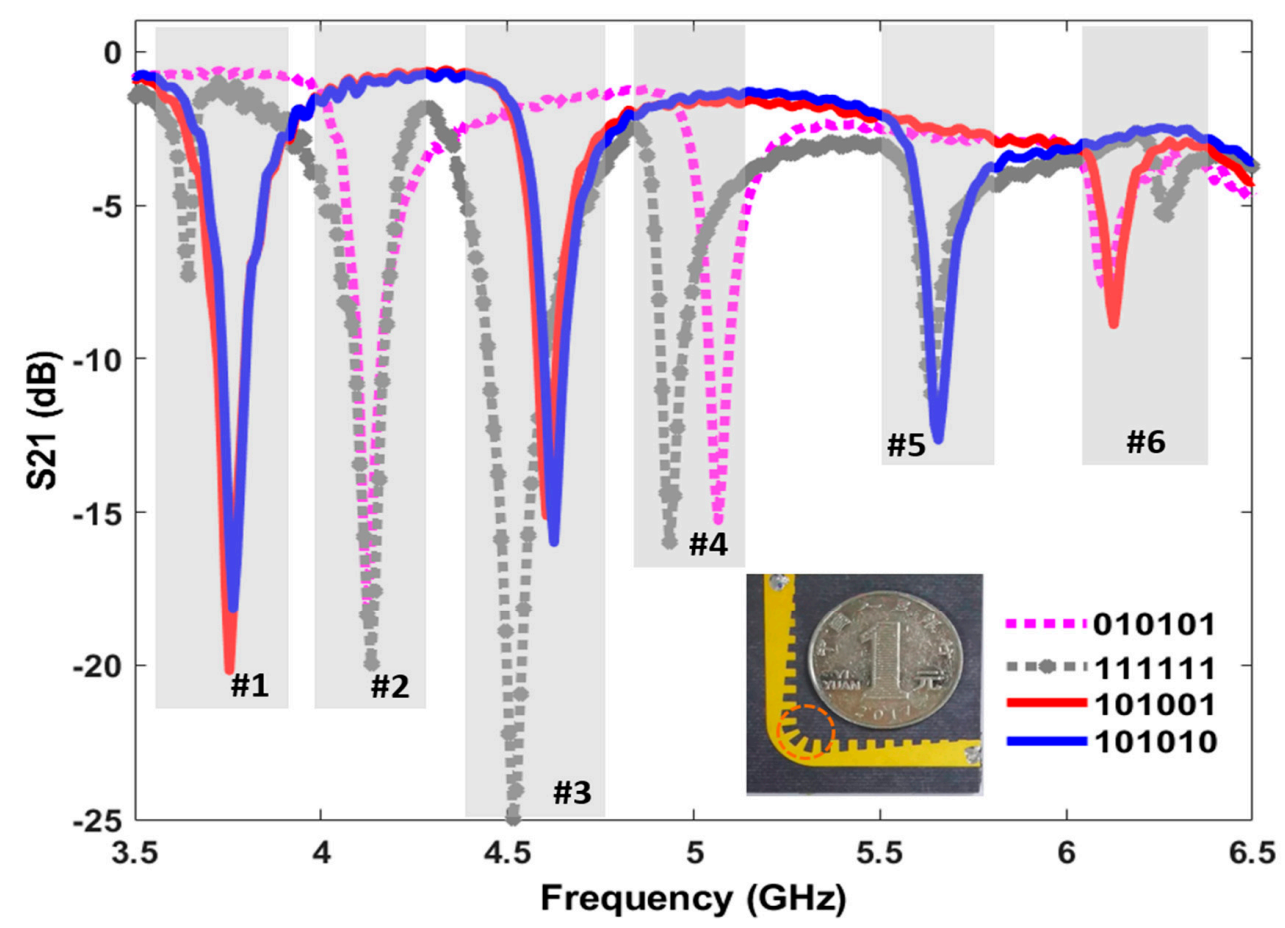

Figure 7. Measured transmission coefficients (S21) with different band rejections and coding sequences. Subgraph: Top side of the fabricated prototype, and the spirals are located in the dashed orange circle in the ground. The numbers \#1-6 represent Gap \#1-6.

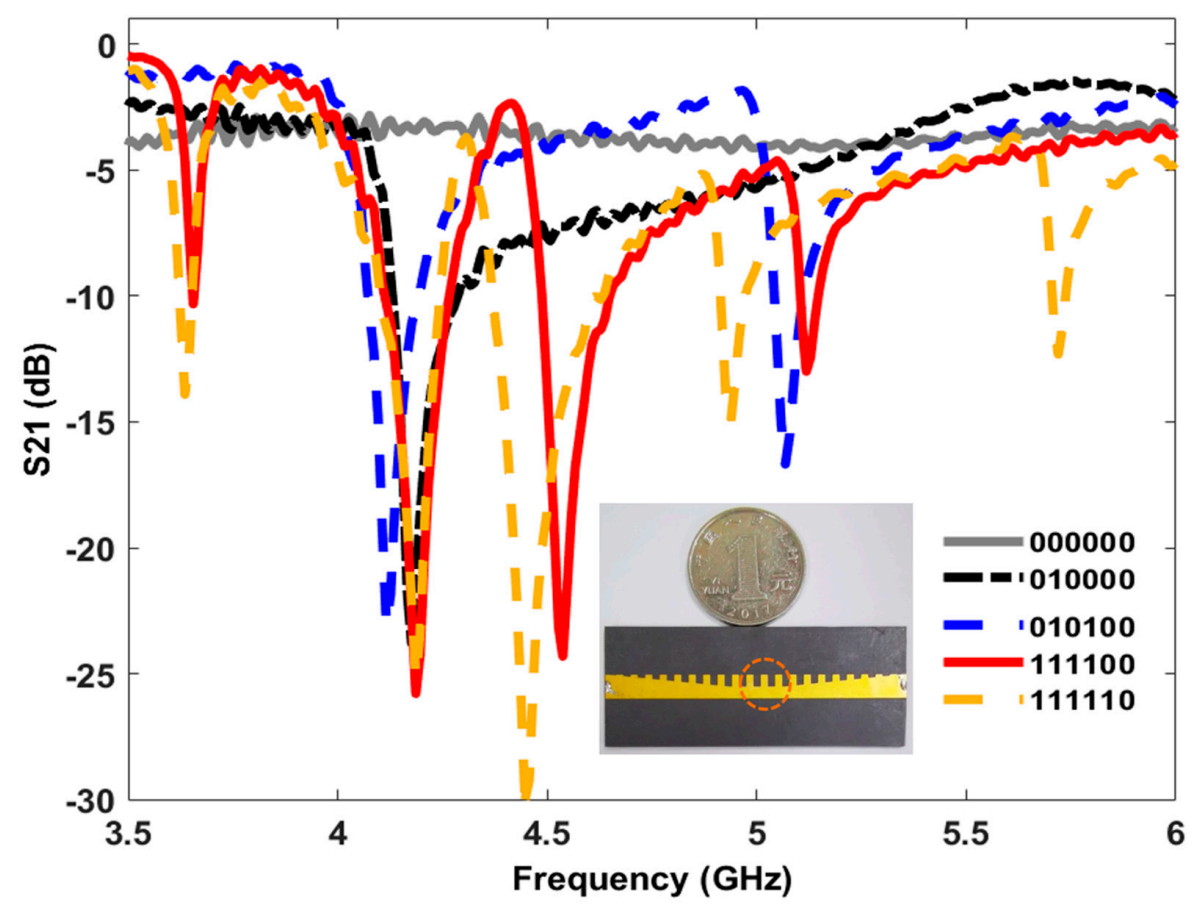

Figure 8. Measured transmission coefficients (S21) with different coding sequences for the straight SSPP TL. Subgraph: Top side of the fabricated prototype, and the spirals are located in the dashed orange circle in the ground. 
The compact multi-band rejection and frequency coding component is also applicable in the straight SSPP circuits, as is demonstrated in Figure 8. The circuit is $63 \mathrm{~mm}$ long in total, including the microstrip lines at the input and output, the transition sections, and a $19.5 \mathrm{~mm}$ section of straight SSPP TL. Five coding sequences are designed and verified in Figure 8. It is remarkable that the solid grey curve, which presents the 000000 code when there is no gap in any spiral branch, is between -3 to $-4 \mathrm{~dB}$. The reduction of S21 is mainly due to the influence of a dominant resonance at about $3.05 \mathrm{GHz}$ (which is not plotted in the figure). From the measured S21 curves, the five different coding sequences can be recognized clearly and correctly.

\section{Discussion}

\subsection{Resolution and Sensitivity}

In the experiment, an example of the proposed 6-bit coding element has been verified. Each resonance frequency is independently designable in accordance with the position of the gap in each branch. In this design, the coordinate of the gap in one branch is defined using the helix equation in the local coordinates whose origin is the center of the spiral as:

$$
x=1.8 r \cos (\pi r), y=1.8 r \sin (\pi r), r=\frac{\theta}{360^{\circ}}\left(\text { in } \mathrm{mm}, 0<\theta<1080^{\circ}\right) \text {. }
$$

when $\theta$ increases from $0^{\circ}$, the gap moves from the center of the spiral to the far end of the branch, with the effective length of the branch and the resonating current in it increasing from 0 accordingly. Figure 9 a demonstrates that the resonance frequency of one branch can be finely manipulated when $\theta$ in Equation (1) varies. Generally, the larger value of $\theta$, which indicates the longer resonating current, brings in the lower resonance frequency. Another demonstration is carried out that if we fix five of the six gaps and slide the last one (e.g., Gap \#2), the resonance frequency on the flexible branch is also controlled smoothly without influencing the resonances in other branches, as is shown in Figure 9b. Therefore, we conclude that the six resonance frequencies can be finely and independently tuned. In addition, it is also observed from Figure 9 that the higher-frequency resonance has the lower quality factor. In other words, when a branch is cut off at the near end and the induced current is short in length, the corresponding resonance is weak and the quality factor is depressed.
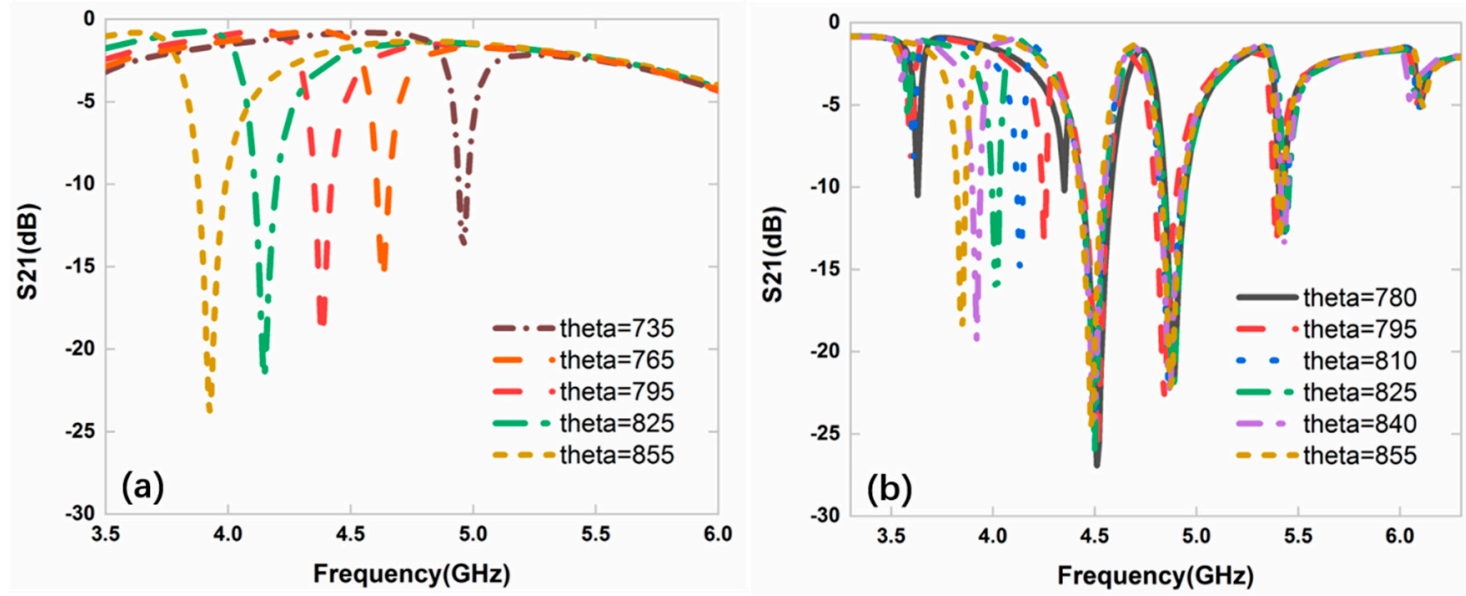

Figure 9. (a) The resonance frequency of one branch when the position of the gap is changed with a varying $\theta$. (b) The resonance frequency of one branch when the position of the gap is changed with a varying $\theta$, meanwhile, the gaps in other branches are fixed.

When the complementary spiral resonator departs from the TL, as is indicated in Figure 10, the resonance frequencies remain nearly unchanged, but the quality factors of the resonances decrease. It is due to the fact that the resonance of the spiral is excited by the 
EM fields in the TL, and most energy of the EM field is localized in a subwavelength scale around the TL. In view of this, the proposed spiral resonator is sensitive to its position in the circuit.

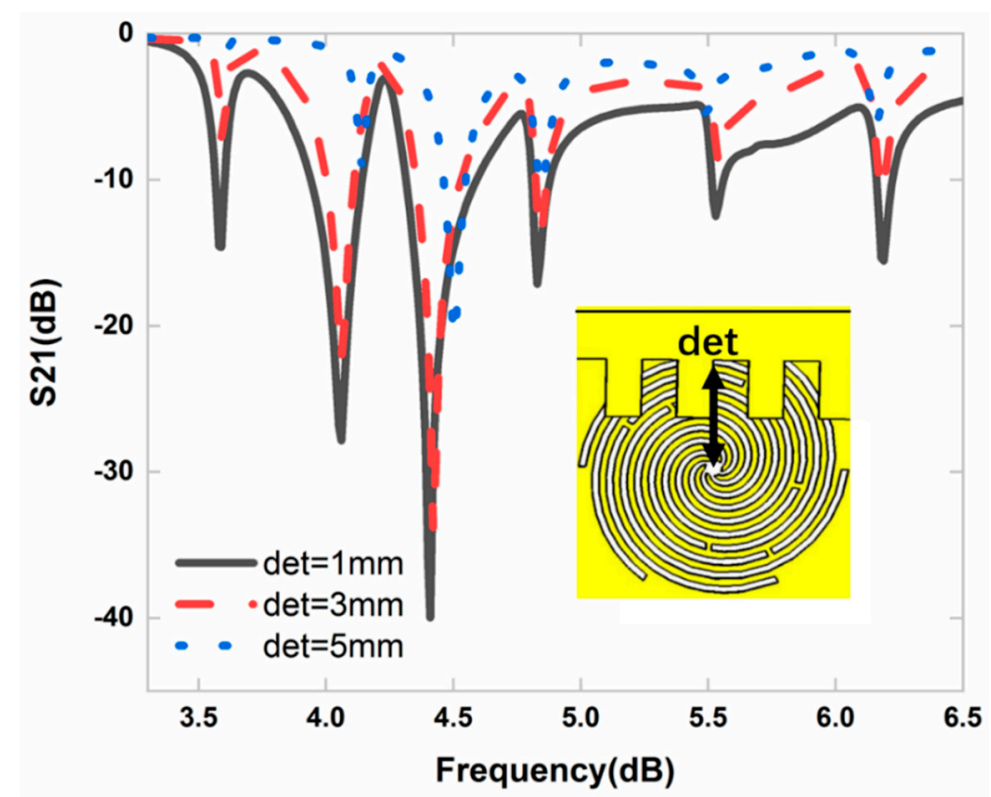

Figure 10. Resonances of the 6-bit coding element when the spiral deviates from the straight TL.

In fact, the quality factor is vital to the maximum number of branches in one spiral resonator, because a resonance with a low quality factor may not be recognized correctly in the frequency domain. Increasing the length and width of branches, as well as decreasing the thickness of the substrate, is helpful to enhance the resonance. Another limitation to the maximum number of bit is the reliable widths of line and gap in the technique of printed circuit board (PCB). As long as the fabrication precision is allowed, more and longer branches can be included in one spiral to present more bits. However, when the branches are located much too tightly, the EM field in one branch may excite the resonance in other branches, and two or more branches will resonate together. Therefore, there may be a tradeoff between the size of the spiral and the maximum number of bits. We predict that higher bit density is achievable for this kind of coding component, and more investigation is needed in the future work.

\subsection{Applicability in the MS Circuit}

The proposed complementary spiral resonator is also applicable in other types of circuits provided it is effectively excited. For example, when the resonator is located right below the straight MS line (please refer to the inset of Figure 11), the six resonances are found again and the resonance frequencies are close to those given in Figure 10 when the straight grounded SSPP TL is used. In view of this, we conclude that the resonant performance of the complementary spiral resonator is independent with the SSPP structure. However, propagating performance of the MS circuit decreases significantly when there exists bending or protuberance because a big portion of energy radiates to the environment. In Figure 11, it is observed that when the MS circuit contains a $90^{\circ}$ bend, the EM field in the TL cannot excite the resonator effectively, and as a result the six resonances start to merge. In contrast, both the straight grounded SSPP TL and the bending one can effectively excite the spiral with comparable intensity. In view of this, we remark that the proposed component in the plasmonic circuit is especially suitable for integrated systems with complicated environments and flexible layouts. 


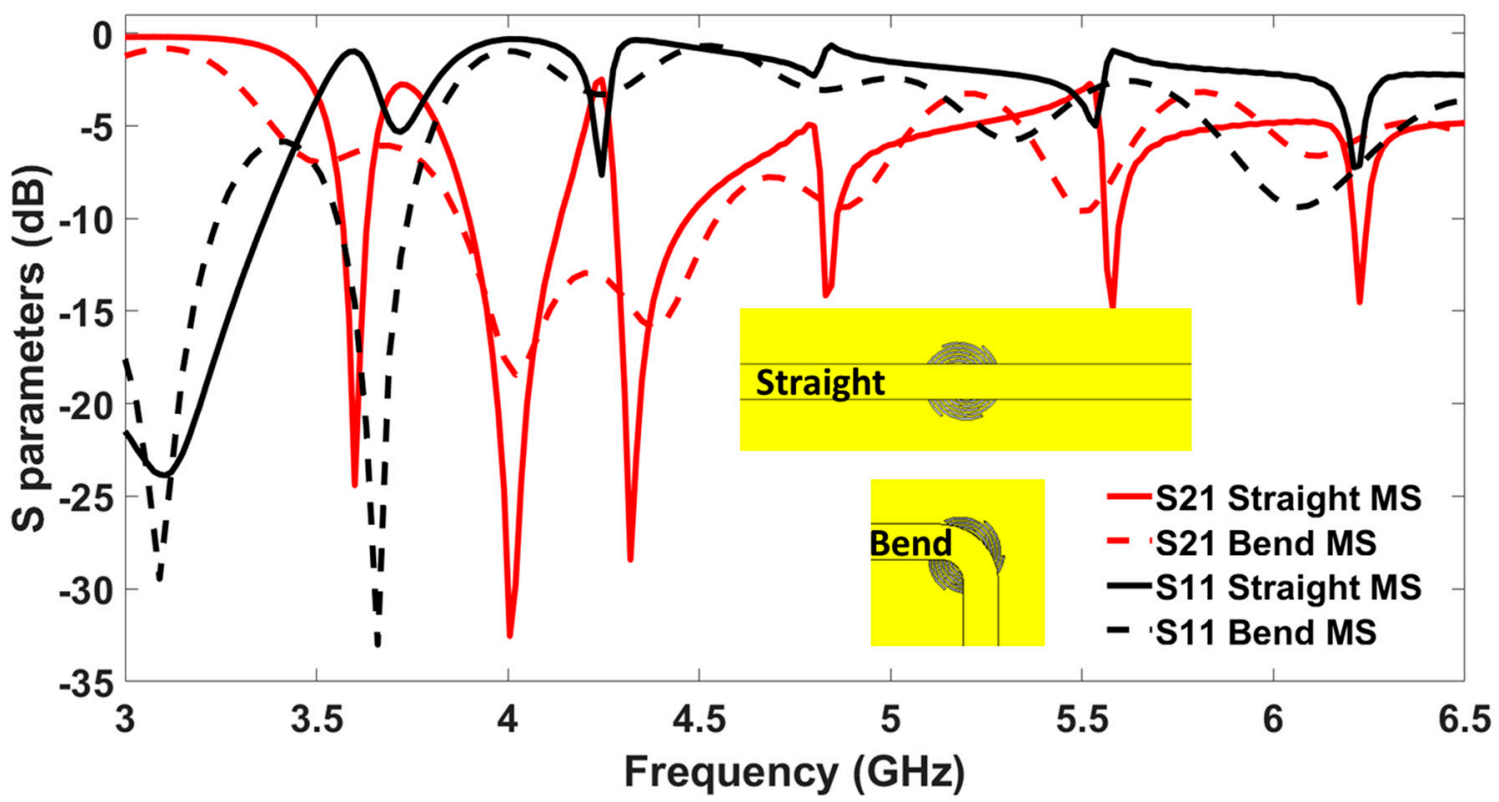

Figure 11. Simulated transmission coefficients (S21) and reflection coefficients (S11) when the complementary spiral resonator is excited by the straight MS line and the bending one.

\section{Conclusions}

In this work, we propose a complementary six-branch spiral resonator that is able to provide independent and flexible resonance in each single branch. In this way, controllable multi-band rejection can be realized in compact circuits. From the point of view of digital coding metamaterials, the multi-band rejection reveals a kind of 6-bit digital coding in frequency domain, where " 1 " represents the existence of rejection in a specific band, whilst " 0 " represents the inexistence of rejection in the band. Simulated and measured results have demonstrated this design, and more or less branches may be adopted to realize more or less bits.

This type of resonator is electrically small and could be easily sculptured in the ground of a transmission line without occupying extra space. It can be embedded in plasmonic circuits and systems to realize frequency identification, e.g., to transmit information encoded in the frequency spectrum. In addition, the proposed complementary six-branch spiral can also be used to detect the change of surrounding medium, and therefore has good potentials in high-resolution sensing and locating.

Author Contributions: Conceptualization, W.T. and T.J.C.; methodology, W.T.; investigation, Y.H.; measurement, W.T.; writing—original draft preparation, W.T. and Y.H.; writing—review and editing, T.J.C.; funding acquisition, W.T. and T.J.C. All authors have read and agreed to the published version of the manuscript.

Funding: This work was supported in part from the National Science Foundation of China under Grant Nos. 61631007 and 61971134, in part from the 111 Project under Grant No. 111-2-05, and in part from the Fundamental Research Funds for the Central Universities under Grant No. 2242020R40079.

Acknowledgments: The authors thank Xuanru Zhang for her suggestion and help during the revision of this manuscript.

Conflicts of Interest: The authors declare no conflict of interest.

\section{References}

1. Pendry, J.B.; Holden, A.J.; Stewart, W.J.; Youngs, I. Extremely low frequency plasmons in metallic mesostructures. Phys. Rev. Lett. 1996, 76, 4773. [CrossRef] [PubMed]

2. Pendry, J.B.; Holden, A.J.; Robbins, D.J.; Stewart, W.J. Magnetism from conductors and enhanced nonlinear phenomena. IEEE Trans. Microw. Theory Technol. 1999, 47, 2075. [CrossRef] 
3. Schurig, D.; Mock, J.J.; Smith, D.R. Electric-field-coupled resonators for negative permittivity metamaterials. Appl. Phys. Lett. 2006, 88, 041109. [CrossRef]

4. Liu, R.; Ji, C.; Mock, J.J.; Chin, J.Y.; Cui, T.J.; Smith, D.R. Broadband ground-plane cloak. Science 2009, 323, 366-369. [CrossRef] [PubMed]

5. Liu, R.; Cui, T.J.; Huang, D.; Zhao, B.; Smith, D.R. Description and explanation of electromagnetic behaviors in artificial metamaterials based on effective medium theory. Phys. Rev. E 2007, 76, 026606. [CrossRef] [PubMed]

6. Shelby, R.; Smith, D.R.; Schultz, S. Experimental verification of a negative index of refraction. Science 2001, 292, 77. [CrossRef] [PubMed]

7. Schurig, D.; Mock, J.J.; Justice, B.J.; Cummer, S.A.; Pendry, J.B.; Starr, A.F.; Smith, D.R. Metamaterial electromagnetic cloak at microwave frequencies. Science 2006, 314, 977. [CrossRef]

8. Smith, D.R.; Mock, J.J.; Starr, A.F.; Schurig, D. Gradient index metamaterials. Phys. Rev. E 2005, 71, 036609. [CrossRef]

9. Ma, H.F.; Cui, T.J. Three-dimensional broadband and broad-angle transformation-optics lens. Nat. Commun. 2010, 1, 124. [CrossRef]

10. Ma, H.F.; Cui, T.J. Three-dimensional broadband ground-plane cloak made of metamaterials. Nat. Commun. 2010, 1, 21. [CrossRef]

11. Baena, J.D.; Bonache, J.; Martin, F.; Sillero, R.M.; Falcone, F.; Lopetegi, T.; Laso, M.A.G. Equivalent-circuit models for split-ring resonators and complementary split-ring resonators coupled to planar transmission lines. IEEE Trans. Microw. Theory Technol. 2005, 53, 1451. [CrossRef]

12. Falcone, F.; Lopetegi, T.; Laso, M.A.G.; Baena, J.D.; Bonache, J.; Beruete, M.; Marques, R.; Martin, F.; Sorolla, M. Babinet principle applied to the design of metasurfaces and metamaterials. Phys. Rev. Lett. 2004, 93, 197401. [CrossRef] [PubMed]

13. Yu, N.; Genevet, P.; Kats, M.A.; Aieta, F.; Tetienne, J.; Capasso, F.; Gaburro, Z. Light propagation with phase discontinuities: Generalized laws of reflection and refraction. Science 2011, 334, 333. [CrossRef] [PubMed]

14. Sun, S.; He, Q.; Xiao, S.; Xu, Q.; Li, X.; Zhou, L. Gradient-index meta-surface as a bridge linking propagating waves and surface waves. Nat. Mater. 2012, 11, 426. [CrossRef] [PubMed]

15. Cui, T.J.; Qi, M.Q.; Wan, X.; Zhao, J.; Cheng, Q. Coding metamaterials, digital metamaterials, and programmable metamaterials. Light 2014, 3, e218. [CrossRef]

16. Cui, T.J.; Liu, S.; Li, L. Information entropy of coding metasurface. Light 2016, 5, e16172. [CrossRef]

17. Zhang, L.; Chen, X.Q.; Shao, R.W.; Dai, J.Y.; Cheng, Q.; Castaldi, G.; Galdi, V.; Cui, T.J. Breaking reciprocity with space-time-coding digital metasurfaces. Adv. Mater. 2019, 31, 1904069. [CrossRef]

18. Dai, J.Y.; Tang, W.K.; Zhao, J.; Li, X.; Cheng, Q.; Ke, J.C.; Chen, M.Z.; Jin, S.; Cui, T.J. High-speed wireless communications through a simplified architecture based on time-domain digital coding metasurface. Adv. Mater. Tech. 2019, 4, 1900044. [CrossRef]

19. Maier, S.A. Plasmonics: Fundamentals and Applications, 1st ed.; Springer: Berlin/Heidelberg, Germany, 2010 ; pp. $21-37$.

20. Pendry, J.B.; Martin-Moreno, L.; Garcia-Vidal, F.J. Mimicking surface plasmons with structured surfaces. Science 2004, $305,847$. [CrossRef]

21. García-Vidal, F.J.; Martín-Moreno, L.; Pendry, J.B. Surfaces with holes in them: New plasmonic metamaterials. J. Opt. A 2005, 7, S97-S101. [CrossRef]

22. Fernandez-Dominguez, A.I.; Martin-Moreno, L.; Garcia-Vidal, F.J.; Andrews, S.R.; Maier, S.A. Spoof surface plasmon polariton modes propagating along periodically corrugated wires. IEEE J. Sel. Top. Quant. Electron. 2008, 14, 1515. [CrossRef]

23. Lockyear, M.J.; Hibbins, A.P.; Sambles, J.R. Microwave surface-plasmon-like modes on thin metamaterials. Phys. Rev. Lett. 2009, 102, 1-4. [CrossRef] [PubMed]

24. Yu, N.; Capasso, F. Wavefront engineering for mid-infrared and terahertz quantum cascade lasers [Invited]. J. Opt. Soc. Am. B 2010, 27, B18-B35. [CrossRef]

25. Naik, G.V.; Shalaev, V.M.; Boltasseva, A. Alternative plasmonic materials: Beyond gold and silver. Adv. Mater. 2013, 25, 3264. [CrossRef] [PubMed]

26. Rusina, A.; Durach, M.; Stockman, M.I. Theory of spoof plasmons in real metals. Appl. Phys. A 2010, 100, 375. [CrossRef]

27. Gric, T. Spoof plasmons in corrugated transparent conducting oxides. J. Electromag. Waves Appl. 2016, 30, 721. [CrossRef]

28. Shen, X.; Cui, T.J.; Martin-Canob, D.; Garcia-Vidal, F.J. Conformal surface plasmons propagating on ultrathin and flexible films. Proc. Natl. Acad. Sci. USA 2013, 110, 40-45. [CrossRef]

29. Shen, X.; Cui, T.J. Ultrathin plasmonic metamaterial for spoof localized surface plasmons. Laser Photon. Rev. 2014, 8, 137. [CrossRef]

30. Zhang, H.C.; Zhang, Q.; Liu, J.F.; Tang, W.; Fan, Y.; Cui, T.J. Smaller-loss planar SPP transmission line than conventional microstrip in microwave frequencies. Sci. Rep. 2016, 6, 23396. [CrossRef]

31. Zhang, H.C.; Cui, T.J.; Zhang, Q.; Fan, Y.; Fu, X. Breaking the challenge of signal integrity using time-domain spoof surface plasmon polaritons. ACS Photonics 2015, 2, 1333-1340. [CrossRef]

32. Zhang, H.C.; Fan, Y.; Guo, J.; Fu, X.; Li, L.; Qian, C.; Cui, T.J. Secondharmonic generation of spoof surface plasmon polaritons using nonlinear plasmonic metamaterials. ACS Photonics 2016, 3, 139. [CrossRef]

33. Wang, M.; Ma, H.F.; Tang, W.X.; Zhang, H.C.; Wang, Z.X.; Cui, T.J. Surface plasmon polaritons to reach reconfigurable plasmonic devices. Adv. Mater. Tech. 2019, 4, 1800603. [CrossRef]

34. Liang, Y.; Yu, H.; Feng, G.Y.; Apriyana, A.A.A.; Fu, X.J.; Cui, T.J. An energy-efficient and low-crosstalk sub-THz I/O by surface plasmonic polariton interconnect in CMOS. IEEE Trans. Microw. Theory Tech. 2017, 65, 2762-2774. [CrossRef] 
35. Zhang, H.C.; Zhang, L.P.; He, P.H.; Xu, J.; Qian, C.; Garcia-Vidal, F.J.; Cui, T.J. A plasmonic route for integrated wireless communication of sub-diffraction-limited signals. Light 2020, 9, 113. [CrossRef]

36. Ma, H.F.; Shen, X.P.; Cheng, Q.; Jiang, W.X.; Cui, T.J. Broadband and high-efficiency conversion from guided waves to spoof surface plasmon polaritons. Laser Photonics Rev. 2014, 8, 146. [CrossRef]

37. Kianinejad, A.; Chen, Z.N.; Qiu, C. Design and modeling of spoof surface plasmon modes-based microwave slow-wave transmission line. IEEE Trans. Microw. Theory Tech. 2015, 63, 1817. [CrossRef]

38. Pozar, D.M. Microwave Engineering, 2nd ed.; John Wiley and Sons, Inc.: New York, NY, USA, 2006; pp. $422-443$.

39. Gao, Z.; Wu, L.; Gao, F.; Luo, Y.; Zhang, B.L. Spoof plasmonics: From metamaterial concept to topological description. Adv. Mater. 2018, 30, 1706683. [CrossRef]

40. Zhang, W.; Zhu, G.; Sun, L.; Lin, F. Trapping of surface plasmon wave through gradient corrugated strip with underlayer ground and manipulating its propagation. Appl. Phys. Lett. 2015, 106, 021104. [CrossRef]

41. Liang, Y.; Yu, H.; Zhang, H.C.; Yang, C.; Cui, T.J. On-chip sub-terahertz surface plasmon polariton transmission lines in CMOS. Sci. Rep. 2015, 5, 14853. [CrossRef]

42. Lee, R.; Wang, B.; Cappelli, M.A. Plasma modification of spoof plasmon propagation along metamaterial-air interfaces. Appl. Phys. Lett. 2017, 111, 261105. [CrossRef]

43. Joy, S.R.; Erementchouk, M.; Yu, H.; Mazumder, P. Spoof plasmon interconnects-communications beyond RC limit. IEEE Trans. Commun. 2019, 67, 599. [CrossRef]

44. Zhang, X.R.; Tang, W.X.; Zhang, H.C.; Xu, J.; Bai, G.D.; Liu, J.F.; Cui, T.J. A spoof surface plasmon transmission line loaded with varactors and short-circuit stubs and its application in Wilkinson power dividers. Adv. Mater. Technol. 2018, 3, 1800046. [CrossRef]

45. Wang, M.; Tang, M.; Zhang, H.C.; Zhang, L.P.; Cui, T.J.; Mao, J. Crosstalk noise suppression between single and differential transmission lines using spoof surface plasmon polaritons. IEEE Trans. Compon. Packg. Manuf. Technol. 2020, 10, 1367. [CrossRef]

46. Baena, J.D.; Marques, R.; Medina, F.; Martel, M. Artificial magnetic metamaterial design by using spiral resonators. Phys. Rev. B 2004, 69, 14402. [CrossRef]

47. Cui, T.J.; Tang, W.X.; Yang, X.M.; Mei, Z.L.; Jiang, W.X. Metamaterials-Beyond Crystals, Noncrystals, and Quasicrystals, 1st ed.; CRC Press: Boca Raton, FL, USA, 2016; pp. 29-42.

48. Zhang, X.R.; Zhang, H.C.; Tang, W.X.; Liu, J.F.; Fang, Z.; Wu, J.W.; Cui, T.J. Loss analysis and engineering of spoof surface plasmons based on circuit topology. IEEE Antennas Wirel. Propag. Lett. 2017, 16, 3204. [CrossRef]

49. Tang, W.X.; Zhang, H.C.; Liu, J.F.; Xu, J.; Cui, T.J. Reduction of radiation loss at small-radius bend using spoof surface plasmon polariton transmission line. Sci. Rep. 2017, 7, 41077. [CrossRef] 